To appear in the International Journal of Computer Mathematics Vol. 00, No. 00, Month 20XX, 1-20

\title{
Natural Gas Storage Valuation and Optimisation under Time-inhomogeneous Exponential Lévy Processes
}

\author{
N. Safarov* and C. Atkinson \\ Department of Mathematics, Imperial College London, London, UK \\ ( October 2015)
}

\begin{abstract}
We analyse a stochastic control problem for the valuation of a natural gas storage facility while taking into account operating characteristics. The underlying natural gas spot price dynamics is assumed to follow a time-inhomogeneous exponential Lévy process [6]. This underlying incorporates common features of gas spot prices such as seasonality, mean-reversion and price spikes with seasonal jump intensities. The corresponding HJB equation is the non-linear PIDE which is solved by an explicit finite difference method. The numerical approach gives us both the value of the storage contract and optimal operating strategy depending on the gas price, current level of gas in the storage and time. The effects of seasonality and seasonal jump intensity are investigated by observing the surfaces of control strategies and contract values.
\end{abstract}

Keywords: gas storage; stochastic control; HJB equation; independent increment process; mean-reversion

2010 AMS Subject Classification: 35Q93; 35R09; 49M25; 60G51; 65C20

\section{Introduction}

The demand for natural gas has grown significantly in recent years. The development of gas-fired electricity generation further contributes to increasing demand. Gas storage provides an efficient and reliable way to deal with the demand-supply fluctuations. Gas is injected to the storage during low demand periods and released when the demand is high. The variations in gas demand are associated with increased gas consumption for heating and gas-fired power generators during winter and summer months, respectively. Operating characteristics and the random nature of natural gas spot prices are two main factors that need to be incorporated into the valuation and optimisation problems of a gas storage facility.

The main operating characteristics of natural gas storage facilities are: base and working gas capacities, deliverability, injection capacity, and cycling. The amount of permanent gas required to provide adequate reservoir pressure is the base gas, while working gas capacity is the amount of gas available for production and sale. The rate at which the reservoir releases gas from its reserves is deliverability and it is highest (lowest) when the reservoir is closest to its maximum capacity ( nearly empty). The rate at which natural gas can be pumped into storage for later use is called injection capacity. The injection rate decreases as the reservoir approaches its maximum and reaches its highest value when the reservoir is empty. Cycling is the number of times that the working gas volumes can be pumped and released in a year. As single cycle storage facilities are

\footnotetext{
*Corresponding author. Email: nematsafarov88@gmail.com
} 
not efficient, high deliverability multiple cycle (HDMC) facilities are used to meet both summer and winter demand.

Depending on their operating characteristics we can differentiate three types of natural gas storage facilities:depleted oil and gas reservoirs, salt cavern storage, and aquifer storage. All of these facilities have their own advantages and disadvantages. In this work we concentrate on salt cavern facilities as they are HDMC and require very low base gas. For more details regarding the other two types and their operating characteristics please refer to [26].

The precise valuation and optimisation of a gas storage facility depends not only on its operating characteristics but also on the random dynamics of natural gas spot prices. In other words, investment and control decisions related to the gas storage have to be made under uncertainty of future spot prices. Real options theory can be applied to model this decision making process (see [15]). The real options theory approaches investment decisions from the point of view of derivatives on financial assets. In the absence of operational constraints the gas storage problem can be viewed as a series of call and put options of different strikes. If we consider operating characteristics as well, then the storage facility becomes more complicated because of the opportunity costs. More precisely, when gas is released (injected) both the amount and the rate of future gas release (injection) are decreased. But the dependence between the opportunity costs and the amount of gas in the reservoir is nonlinear. Numerical valuation and control become reasonably complicated if we investigate these issues coupled with stochastic dynamics of natural gas prices. We will use numerical partial differential equations (PDE) technique for the reasons described in [26].

In this paper we are investigating the natural gas storage model presented in [26]. Our main contribution is the adaptation of this model to a spot price with more complicated dynamics, namely time-inhomogeneous exponential Lévy process. This underlying incorporates common features of gas spot prices such as seasonality, mean-reversion and price spikes with seasonal intensities. We then extend the explicit finite difference method proposed by [25] to solve the resulting non-linear partial integral differential equation (PIDE). Based on this approach, a special example for a storage facility has been numerically implemented.

\section{Literature review}

In practice there are several approaches for gas storage valuation. The two most popular ones are the classical intrinsic valuation based on physical futures contracts and the extrinsic valuation, which uses spot trading strategies. Intrinsic valuation assumes that the demand for natural gas is seasonal, with peaks during winter and lows during summer. The storage strategy is determined by exploiting the predictable seasonal shape of the natural gas futures curve. In other words, the storage owner decides to buy/sell futures at the beginning of the storage contract and receives/delivers natural gas at the expiration of futures. The optimal futures positions is determined by solving a linear optimization problem. But the storage owner can't benefit from the futures curve movements as the initial optimal futures position is kept unchanged throughout the whole contract duration (see [17]). [18] extends this static approach to the rolling intrinsic in order to take advantage of the changing dynamics of the futures prices.

The fundamental idea behind these two futures-based approaches is to buy cheap summer futures and sell expensive winter futures. Therefore, the storage value depends on the seasonal spread between cold and warm periods of the year. However, the seasonal 
spread between winter and summer prices has declined in recent years due to the surge in shale gas supply and extensive use of natural gas in power generation. This fall diminishes intrinsic value of a gas storage contract and makes futures based valuation methods less reliable. These seasonality issues can be handles by using natural gas spot price based extrinsic valuation techniques.

Modelling of the underlying natural gas futures curve or spot price process has become an outstanding issue in the recent years. A mean-reverting process is a common model for the spot price. For example, [24] presented 3 mean reverting models for commodity spot prices while assuming that stochastic convenience yield and interest rates also mean revert. [9] used the Least Square Monte Carlo method (initially proposed by [22] for American options) for storage valuation, while assuming the following one factor model for the spot price, which is calibrated to the initial futures curve:

$$
\frac{d S(t)}{S(t)}=\kappa(\mu(t)-\ln S(t)) d t+\sigma d B(t),
$$

where $B$ is a standard Brownian motion, $\mu$ is a time-dependent parameter, calibrated to the initial futures curve $(F(0, T))_{T \geq 0}$, provided by the market; the mean reversion parameter $\kappa$ and the volatility $\sigma$ are positive constants. One factor models are popular because they simplify the theoretical and numerical work required to solve storage problems. As it is stated in $[8,20]$, it's unrealistic to assume that one factor models can capture all characteristics of highly volatile natural gas prices. [23] extended (1) to the two-factor mean-reverting model where the long-term mean is also a mean-reverting process. [10] extended the application of the Least Square Monte Carlo method to multi-factor spot processes.

Another way to model the natural gas spot process is to consider it as the limit $S(t)=\lim _{T \rightarrow t} F(t, T)$ (see [27]), where the futures curve is given by n-factor log-normal dynamics:

$$
\frac{d F(t, T)}{F(t, T)}=\sum_{i=1}^{n} \sigma_{i}(t) e^{-a_{i}(T-t)} d B_{t}^{i}
$$

By continuity of $F(t, T)$ we get $S(t)=F(t, t)$. The purpose of storage facilities is to mitigate seasonal demand and price fluctuations in the natural gas market by purchasing and injecting gas into storage during the low demand periods and releasing gas reserves during the high demand months. But the ability of gas storage to mitigate demand fluctuations is not perfect because of the increased use of gas as the fuel for power generation. These market imperfections can cause sudden price spikes far outside normal seasonal equilibrium levels. These spikes can be understood as the market signals for more investment in storage capacity. Therefore, price spikes have to be included to our spot price models as they carry out significant arbitrage opportunities.

However, none of the models described above exhibits spiky behavior of natural gas spot prices. The first notion of jumps within gas storage valuation was proposed by [26] where the risk adjusted price $(P)$ is modelled by the following one dimensional continuous time Markov process:

$$
d P=\mu_{1}(P, t) d t+\sigma_{1}(P, t) d X_{1}+\sum_{k=1}^{N} \gamma_{k}\left(P, t, J_{k}\right) d q_{k}
$$


where $\mu, \sigma$, and the $\gamma_{k}$ 's are any arbitrary functions and the $J_{k}$ s are arbitrary jump sizes with distributions $Q_{k}(J) . X_{1}$ and $d q_{k}$ 's denote the standard Brownian motion and Poisson processes respectively. (2) covers a wide range of spot price models for natural gas and is tractable for numerical calculations. However, it's jump component isn't appropriate to represent instant and mean-reverting spikes of gas prices.

[4] proposes a more realistic alternative by defining energy spot dynamics as a sum of Ornstein-Uhlenbeck processes with different mean-reverting speeds. The only sources of randomness of the spot prices are positive pure jump processes. The additive structure of the model makes it difficult to derive futures curves. Therefore, [2] considers a stochastic volatility model by [1] for equity markets in the context of energy commodities. [7] generalises this non-Gaussian approach to a multivariate case to model the dynamics of cross-commodity spot prices.

The spiky behavior of energy prices can differ significantly between cold and warm months. Hence, time-inhomogeneous jump processes should be applied to capture these seasonality effects. [6] describes the dynamics of electricity prices in the following way:

$$
\ln S(t)=\ln \Lambda(t)+\sum_{i=1}^{m} X_{i}(t)+\sum_{j=1}^{n} Y_{j}(t),
$$

where

$$
\begin{aligned}
d X_{i}(t) & =\left(\mu_{i}(t)-\alpha_{i}(t) X_{i}(t)\right) d t+\sum_{k=1}^{p} \sigma_{i k}(t) d B_{k}(t), \\
d Y_{j}(t) & =\left(\delta_{j}(t)-\beta_{j}(t) Y_{j}(t)\right) d t+\eta_{j}(t) d L_{j}(t)
\end{aligned}
$$

for $i=1, \ldots, m$ and $j=1, \ldots, n$. $L_{j}$ 's are time-inhomogeneous Lévy processes, while the coefficients $\mu_{i}, \alpha_{i}, \delta_{j}, \beta_{j}, \eta_{j}$ are continuous functions of t.

In practice, gas storage contracts are valued and hedged via heuristic models due to their simplicity in dealing with high-dimensional and multi-factor price dynamics. However, derivation of the optimal injection and withdrawal strategy and inclusion of the physical constraints require the application of a stochastic control framework. This is especially important for HDMC facilities that have far greater flexibility and therefore require more advanced strategies than conventional winter withdrawal and summer refilling. Application of the dynamic programming theory translates the stochastic optimisation problem into the Hamilton-Jacobi-Bellman (HJB) equation. The resulting PDEs can be solved by means of standard numerical techniques.

[26] developed a stochastic control model for natural gas storage facilities while assuming these spot prices follow (2). The HJB PIDE is then solved via an explicit finite difference method known as total variation diminishing scheme. [13] implements fully implicit and Crank-Nicolson time-stepping schemes based on a semi-Lagrangian approach and proves the convergence of fully implicit time-stepping to the viscosity solution of the HJB equation in the presence of simple one factor spot price dynamics.

An alternative to the one factor spot price models is the regime-switching framework initially proposed by [19]. A regime-switching model consists of several regimes and the price process can randomly shift between these regimes due to demand-supply fluctuations, political instability, weather changes and other reasons. The gas price follows a distinct stochastic process within each regime. [12] and [14] apply a regime-switching approach to investigate stochastic control problems related to natural gas storage and 
implement the numerical calculation via Monte Carlo and finite difference methods, respectively.

\section{Natural gas spot price}

Let $\left(\Omega, \mathcal{F},\left\{\mathcal{F}_{t}\right\}_{t \geq 0}, \mathbb{P}\right)$ be our complete filtered probability space. We assume that the natural gas price follows a time-inhomogeneous exponential Lévy model defined by eqs. (3) to (5). For simplicity, we take $m=n=p=1$ and

$$
X(0)+Y(0)=\ln S(0)-\ln \Lambda(0)
$$

Then we have the following dynamics for $S(t)$ :

$$
S(t)=\Lambda(t) \exp (X(t)+Y(t))
$$

where

$$
\begin{aligned}
& d X(t)=(\mu(t)-\alpha(t) X(t)) d t+\sigma(t) d B(t) \\
& d Y(t)=(\delta(t)-\beta(t) Y(t)) d t+\eta(t) d L(t)
\end{aligned}
$$

As we stated earlier, $B(t)$ is a standard Brownian motion; $L(t)$ is time-inhomogeneous Lévy process (or independent increment (II) process); $\Lambda(t)$ is a deterministic, positive and continuously differentiable seasonality function and the coefficients are continuous functions of $t$.

The first fundamental theorem of asset pricing implies that the no-arbitrage requirement is equivalent to the existence of at least one equivalent probability measure which turns all the discounted tradable asset processes into local martingales. Meanwhile, according to the second fundamental theorem of asset pricing the equivalent martingale measure is unique if the market is complete. The models introduced for energy spot prices exhibit incompleteness due to their jumps and storage problems of the commodities. Therefore, any measure $\mathbb{Q}$ equivalent to the objective probability $\mathbb{P}$ is risk-neutral, and the underlying asset process does not need to be a martingale with respect to the risk-neutral measure. Then we get the rather wide class of potential pricing measures and some restrictions must be imposed to perform calculations. [6] suggests the use of the Esscher transform, which has been extensively applied in various pricing models related to Lévy processes. The reason why the Esscher transform is an interesting measure change is its structure preserving property. Although an II process loses its semimartingale characteristics after the transformation, the independence of the increments is still preserved.

We can define the Esscher transform for II processes based on [6]. Let $\theta(t)=(\theta \hat{\theta} t), \theta \tilde{(t}))$ be a two dimensional vector of real-valued continuous functions on $[0, T]$. Let's introduce the following stochastic exponential:

$$
Z^{\theta}(t)=\hat{Z}^{\theta}(t) \times \tilde{Z}^{\theta}(t)
$$


where

$$
\begin{aligned}
& \hat{Z}^{\theta}(t)=\exp \left(\int_{0}^{t} \hat{\theta}(u) d B(u)-\frac{1}{2} \int_{0}^{t} \hat{\theta}^{2}(u) d u\right), \\
& \tilde{Z}^{\theta}(t)=\exp \left(\int_{0}^{t} \tilde{\theta}(u) d L(u)-\psi(0, t, \hat{\theta}(\cdot))\right),
\end{aligned}
$$

where $\psi$ is the cumulant function defined by the Lévy-Kintchine representation for the II process $L(t)$ with spot characteristics $(\gamma(t), C(t), l(d t, d z))$ :

$$
\begin{gathered}
\mathbb{E}\left[\exp \left(i \int_{s}^{t} \theta(u) d L(u)\right)\right]=\exp (\psi(s, t, \theta(\cdot))), \\
\psi(s, t, \theta(\cdot))=i \int_{s}^{t} \theta(u) d \gamma(u)-\frac{1}{2} \int_{s}^{t} \theta^{2}(u) d C(u)+ \\
\int_{s}^{t} \int_{\mathbb{R}}\left\{e^{i \theta(u) z}-1-i \theta(u) z \mathbb{1}_{|z| \leq 1}\right\} l(d z, d u) .
\end{gathered}
$$

[6] imposes the following integrability condition on $L(t)$ in order to apply the Esscher transform:

Condition 1 There exists a constant $c$ such that

$$
\int_{s}^{T} \int_{\mathbb{R}}\left\{e^{c z}-1\right\} l(d z, d u)<\infty .
$$

If the condition

$$
\sup _{0 \leq t \leq T}|\tilde{\theta}(t)|<c
$$

holds together with (11), then Itô formula implies that $\tilde{Z}^{\theta}(t)$ is a martingale process (a local martingale with $\left.\mathbb{E}\left[\tilde{Z}^{\theta}(t)\right]=1\right)$. Meanwhile, if $\hat{\theta}(t)$ is continuous and the Novikov condition holds, then $\hat{Z}^{\theta}(t)$ is also a martingale. Therefore, an equivalent probability measure $\mathbb{Q}^{\theta}$ with the Radon-Nikodym derivative $d \mathbb{Q}^{\theta} / d \mathbb{P}$ can be defined as follows:

$$
\frac{d \mathbb{Q}^{\theta}}{d \mathbb{P}}=Z^{\theta}(t)=\hat{Z}^{\theta}(t) \times \tilde{Z}^{\theta}(t)
$$

According to [6], the SDEs of $X(t)$ and $Y(t)$ under $\mathbb{Q}^{\theta}$ become:

$$
\begin{aligned}
& d X(t)=(\mu(t)+\sigma(t) \hat{\theta}(t)-\alpha(t) X(t)) d t+\sigma(t) d B^{\theta}(t), \\
& d Y(t)=(\delta(t)-\beta(t) Y(t)) d t+\eta(t) d \Phi(t, \tilde{\theta}(t))+\eta(t) d \tilde{L}(t),
\end{aligned}
$$

where $\tilde{L}(t)=L(t)-\mathbb{E}_{\mathbb{Q}^{\theta}}[L(t)]$ is a $\mathbb{Q}^{\theta}$-martingale II process and

$$
\Phi(t, \tilde{\theta}(t))=\frac{\partial}{\partial \theta} \phi(0, t, \tilde{\theta}(t))=\mathbb{E}_{\mathbb{Q}^{\theta}}[L(t)]
$$


with $\phi(s, t, \tilde{\theta}(\cdot))=\psi(s, t,-i \tilde{\theta}(\cdot))$. We can imply from the definition of $\psi(10)$ that

$$
d \Phi(t, \tilde{\theta}(t))=d \gamma(t)+\tilde{\theta}(t) d C(t)+\int_{\mathbb{R}} z\left\{e^{\tilde{\theta}(t) z}-\mathbb{1}_{|z| \leq 1}\right\} l(d z, d t) .
$$

If the function $\Phi$ is absolutely continuous with respect to the Lebesgue measure then

$$
d \Phi(t, \tilde{\theta}(t))=\tilde{\Phi}(t, \tilde{\theta}(t)) d t
$$

for a nice behaving function $\tilde{\Phi}$. Let $J(d z, d t)$ and $\tilde{J}(d z, d t)=J(d z, d t)-e^{\tilde{\theta}(t) z} l(d z, d t)$ be the random jump meeasure and compensated random jump measure of the II process $L(t)$ (see [21]). Then the dynamics of $Y(t)$ can be written as

$$
d Y(t)=(\delta(t)+\eta(t) \tilde{\Phi}(t, \tilde{\theta}(t))-\beta(t) Y(t)) d t+\int_{\mathbb{R}} \eta(t) z \tilde{J}(d z, d t),
$$

We can derive the SDE for $S(t)$ by applying Itô's lemma to (6):

$$
d S(t)=\Lambda^{\prime}(t) \exp (X(t)+Y(t)) d t+\Lambda(t) d \exp (X(t)+Y(t))
$$

where

$$
\begin{aligned}
& \Lambda(t) d \exp (X(t)+Y(t))=S\left(t_{-}\right) d X(t)+S\left(t_{-}\right) d Y(t)+\frac{1}{2} S\left(t_{-}\right) d[X, X]_{t}+ \\
& \int_{\mathbb{R}} S\left(t_{-}\right)\left(e^{\eta(t) z}-1-\eta(t) z\right) J(d z, d t)=S\left(t_{-}\right) d X(t)+S\left(t_{-}\right) d Y(t)+\frac{1}{2} S\left(t_{-}\right) d[X, X]_{t}+ \\
& \int_{\mathbb{R}} S\left(t_{-}\right)\left(e^{\eta(t) z}-1-\eta(t) z\right) e^{\tilde{\theta}(t) z} l(d z, d t)+\int_{\mathbb{R}} S\left(t_{-}\right)\left(e^{\eta(t) z}-1-\eta(t) z\right) \tilde{J}(d z, d t)
\end{aligned}
$$

After substituting eqs. (12), (15) and (17) in (16) we get

$$
\begin{array}{r}
\frac{d S(t)}{S\left(t_{-}\right)}=\bar{\chi}(t) d t-(\alpha(t) X(t)+\beta(t) Y(t)) d t+\sigma(t) d B^{\theta}(t)+\int_{\mathbb{R}}\left(e^{\eta(t) z}-1\right) \tilde{J}(d z, d t)= \\
((\beta(t)-\alpha(t)) X(t)-\beta(t)(X(t)+Y(t))+\bar{\psi}(t)) d t+ \\
\sigma(t) d B^{\theta}(t)+\int_{\mathbb{R}}\left(e^{\eta(t) z}-1\right) \tilde{J}(d z, d t)
\end{array}
$$

where

$$
\begin{aligned}
& \bar{\chi}(t)=\frac{\Lambda^{\prime}(t)}{\Lambda(t)}+\frac{1}{2} \sigma^{2}(t)+\mu(t)+\sigma(t) \hat{\theta}(t)+\delta(t)+ \\
& \eta(t) \tilde{\Phi}(t, \tilde{\theta}(t))+\int_{\mathbb{R}}\left(e^{\eta(t) z}-1-\eta(t) z\right) e^{\tilde{\theta}(t) z} l(d z, d t) .
\end{aligned}
$$

We know from (6) that $X(t)+Y(t)=\ln S(t)-\ln \Lambda(t)$. Then 


$$
\begin{array}{r}
\frac{d S(t)}{S\left(t_{-}\right)}=(\bar{\chi}(t)+(\beta(t)-\alpha(t)) X(t)-\beta(t) \ln S(t)+\beta(t) \ln \Lambda(t)) d t+ \\
\sigma(t) d B^{\theta}(t)+\int_{\mathbb{R}}\left(e^{\eta(t) z}-1\right) \tilde{J}(d z, d t)
\end{array}
$$

For simplicity, we can further denote

$$
\bar{\beta}(t)=\beta(t)-\alpha(t), \quad \chi(t)=\frac{\bar{\chi}(t)+\beta(t) \ln \Lambda(t)}{\bar{\beta}(t)} \quad \text { and } \quad \xi(t)=\frac{\beta(t)}{\bar{\beta}(t)},
$$

to get the following model with stochastic mean for the natural gas spot price:

$$
\begin{aligned}
& \frac{d S(t)}{S\left(t_{-}\right)}=\bar{\beta}(t)(\chi(t)+X(t)-\xi(t) \ln S(t)) d t+\sigma(t) d B^{\theta}(t)+\int_{\mathbb{R}}\left(e^{\eta(t) z}-1\right) \tilde{J}(d z, d t) \\
& d X(t)=(\mu(t)+\sigma(t) \hat{\theta}(t)-\alpha(t) X(t)) d t+\sigma(t) d B^{\theta}(t)
\end{aligned}
$$

\section{Control problem}

In this section we introduce the natural gas storage problem defined by [26] and derive the PIDE for the valuation of gas storage contract. The following notation will be used throughout our calculations:

$S(t)$ - underlying spot price per unit of natural gas; $T$ - contract expiry time; $I$ current amount of working gas inventory that varies within $\left[0, I_{\max }\right] ; V(S, I, t)$ - value of the natural gas storage facility at time $t ; c$ - control variable representing the rate of producing gas from $(c>0)$ or injecting gas into $(c<0)$ the gas storage (no action is performed when $c=0) ; c_{\max }(I)$ - maximum deliverability rate of natural gas from the storage defined by

$$
c_{\max }(I)=k_{1} \sqrt{I}
$$

where $k_{1}$ is a positive constant, $c_{\max }(I)>0$ and $c_{\max }(0)=0$ (no gas can be produced if the gas storage is empty); $\left|c_{\min }(I)\right|$ - maximum injection rate of natural gas into the storage given by

$$
c_{\min }(I)=-k_{2} \sqrt{\frac{1}{I+k_{3}}-\frac{1}{k_{4}}},
$$

where $c_{\min }(I)<0$ and $k_{2}, k_{3}, k_{4}$ are positive constants that satisfy $c_{\min }\left(I_{\max }\right)=0$ (no gas injection when the storage is full); $a(I, c)$ - the rate that shows the amount of gas lost during release or injection while there are $I$ units of gas in storage.

Based on the notation given above, the change in $I$ can be defined by the following deterministic equation:

$$
d I=-(c+a(I, c)) d t
$$

We need to find the optimal value $V(S, I, t)$ and optimal operating strategy $c(S, I, t)$ of 
the gas storage facility via the equation proposed in [26]:

$$
V(S, I, t)=\max _{c(S, I, t)} \mathbb{E}_{\mathbb{Q}^{\theta}}\left[\int_{t}^{T} e^{-\rho(\tau-t)}(c-a(I, c)) S d \tau\right]
$$

subject to

$$
c_{\min }(I) \leq c \leq c_{\max }(I) .
$$

Rewriting (23) in the following way will lead to the Bellman equation:

$$
\begin{gathered}
V=\max _{c} \mathbb{E}_{\mathbb{Q}^{\theta}}\left[\int_{t}^{t+d t} e^{-\rho(\tau-t)}(c-a(I, c)) S d \tau+\int_{t+d t}^{T} e^{-\rho(\tau-t)}(c-a(I, c)) S d \tau\right] \\
=\max _{c} \mathbb{E}_{\mathbb{Q}^{\theta}}\left[\int_{t}^{t+d t} e^{-\rho(\tau-t)}(c-a(I, c)) S d \tau+e^{-\rho d t} \int_{t+d t}^{T} e^{-\rho(\tau-(t+d t))}(c-a(I, c)) S d \tau\right] \\
=\max _{c} \mathbb{E}_{\mathbb{Q}^{\theta}}\left[\int_{t}^{t+d t} e^{-\rho(\tau-t)}(c-a(I, c)) S d \tau+e^{-\rho d t} V(S+d S, I+d I, t+d t)\right] .
\end{gathered}
$$

In our case, $S(t)$ has a stochastic mean $X(t)$. Therefore, we apply Itô's formula to $V(S, X, I, t)$ to expand it in Taylor's series:

$$
\begin{aligned}
V=\max _{c} \mathbb{E}_{\mathbb{Q}^{\theta}}\left[(c-a(I, c)) S d t+(1-\rho d t)\left[V+V_{t} d t-(c+a(I, c)) V_{I} d t+\right.\right. \\
V_{S} d S+V_{X} d X+\frac{1}{2} V_{S S} d[S, S]_{t}+\frac{1}{2} V_{X X} d[X, X]_{t}+V_{S X} d[S, X]_{t}+ \\
\left.\int_{\mathbb{R}}\left(V\left(S e^{\eta(t) z}, X, I, t\right)-V(S, X, I, t)-S\left(e^{\eta(t) z}-1\right) V_{S}\right) J(d z, d t)\right],
\end{aligned}
$$

where

$$
d[S, S]_{t}=\sigma^{2}(t) S_{t}^{2} d t, \quad d[X, X]_{t}=\sigma^{2}(t) d t, \quad d[S, X]_{t}=\sigma^{2}(t) S_{t} d t .
$$

If we substitute eqs. (20), (21) and (27) in (26), eliminate all terms that go to zero faster than $d t$, carry out some simplifications and take expectations and divide by $d t$ then we obtain

$$
\begin{gathered}
\max _{c}\left[V_{t}+\frac{1}{2} \sigma^{2}(t) S^{2} V_{S S}+\frac{1}{2} \sigma^{2}(t) V_{X X}+\sigma^{2}(t) S V_{S X}+\bar{\beta}(t)(\chi(t)+X-\right. \\
\left.\xi(t) \ln S) S V_{S}+(\mu(t)+\sigma(t) \hat{\theta}(t)-\alpha(t) X) V_{X}\right)-\rho V+ \\
\lambda(t) \int_{\mathbb{R}}\left(V\left(S e^{\eta(t) z}, X, I, t\right)-V(S, X, I, t)-S\left(e^{\eta(t) z}-1\right) V_{S}\right) e^{\tilde{\theta}(t) z} \bar{l}(d z)+ \\
\left.(c-a(I, c)) S-(c+a(I, c)) V_{I}\right]=0,
\end{gathered}
$$

where $l(d z, d t)=\lambda(t) \bar{l}(z) d t$. Actually, only two terms in (28) contain the control variable $c$. So, our maximisation reduces to

$$
\max _{c}\left[(c-a(I, c)) S-(c+a(I, c)) V_{I}\right]
$$




$$
c_{\min }(I) \leq c \leq c_{\max }(I) .
$$

Therefore, when we have the optimal strategy $c(S, I, t),(28)$ leads to the following PIDE:

$$
\begin{array}{r}
V_{t}+\frac{1}{2} \sigma^{2}(t) S^{2} V_{S S}+\frac{1}{2} \sigma^{2}(t) V_{X X}+\sigma^{2}(t) S V_{S X}+\bar{\beta}(t)(\chi(t)+X- \\
\left.\xi(t) \ln S) S V_{S}+(\mu(t)+\sigma(t) \hat{\theta}(t)-\alpha(t) X) V_{X}\right)-\rho V+ \\
\lambda(t) \int_{\mathbb{R}}\left(V\left(S e^{\eta(t) z}, X, I, t\right)-V(S, X, I, t)-S\left(e^{\eta(t) z}-1\right) V_{S}\right) e^{\tilde{\theta}(t) z} \bar{l}(d z)+ \\
(c-a(I, c)) S-(c+a(I, c)) V_{I}=0,
\end{array}
$$

We can imply from (23) that at time $t=T$ the maturity condition $V(S, X, I, T)=0$ is satisfied. But for stochastic control problems with more general optimisation equations than (23) the maturity condition will be

$$
V(S, X, I, T)=H(S, I, X)
$$

for some function $H$.

The physical characteristics of the storage facility impose the following two conditions on the extreme values of $I$ (see $[26]$ ):

$$
\begin{aligned}
& c+a(I, c) \leq 0 \quad \text { for } \quad I=0 \\
& c+a(I, c) \geq 0 \quad \text { for } \quad I=I_{\text {max }} .
\end{aligned}
$$

According to the first condition gas can't be released when the reservoir is empty and we have to calculate all the derivatives for the values $I \geq 0$. The reverse is true for the second condition. The boundary conditions w.r.t. state variables will be presented in the next section after simplifying the PIDE (30).

\section{Simplifying PIDE before discretisation}

Before the application of numerical methods, we will employ some simplifications and transformations which do not change the nature of the general equation (30). Let's take $\theta=(\hat{\theta}, \tilde{\theta})$ constant. According to [3], the Lévy measure $l(d z, d t)$ transforms to $e^{\tilde{\theta} z} l(d z, d t)$ under $\mathbb{Q}^{\theta}$. If we assume that $L(t)$ is a compound Poisson process then the jump measure $l$ becomes

$$
l(d z, d t)=\lambda(t) P_{J}(d z) d t
$$

where $\lambda(t)$ and $P_{J}(d z)$ are jump intensity and jump-size distribution, respectively. So,

$$
\begin{aligned}
& l_{\mathbb{Q}^{\theta}}(d z, d t)=e^{\tilde{\theta} z} l(d z, d t)=e^{\tilde{\theta} z} \lambda(t) P_{J}(d z) d t= \\
& e^{\tilde{\theta} z} \lambda(t) P_{J}(d z) d t \frac{\int_{\mathbb{R}} e^{\tilde{\theta} z} P_{J}(d z)}{\int_{\mathbb{R}} e^{\tilde{\theta} z} P_{J}(d z)}=\int_{\mathbb{R}} e^{\tilde{\theta} z} \lambda(t) P_{J}(d z) \frac{e^{\tilde{\theta} z} P_{J}(d z)}{\int_{\mathbb{R}} e^{\tilde{\theta} z} P_{J}(d z)} d t,
\end{aligned}
$$


where denoting

$$
\lambda^{\mathbb{Q}}(t)=\int_{\mathbb{R}} e^{\tilde{\theta} z} \lambda(t) P_{J}(d z) \quad \text { and } \quad P^{\mathbb{Q}}(d z)=\frac{e^{\tilde{\theta} z} P_{J}(d z)}{\int_{\mathbb{R}} e^{\tilde{\theta} z} P_{J}(d z)},
$$

implies

$$
l_{\mathbb{Q}^{\theta}}(d z, d t)=\lambda^{\mathbb{Q}}(t) P^{\mathbb{Q}}(d z) d t .
$$

Thus, $L(t)$ is still a compound Poisson process with different intensity and jump-size distribution. The parameter $\theta$ is called a market price of risk where $\hat{\theta}$ and $\tilde{\theta}$ represent market prices of risk caused by normal price fluctuations and spikes, respectively.

After the introduction of the time to maturity $\tau=T-t$, the PIDE (30) becomes

$$
\begin{gathered}
V_{\tau}=\frac{1}{2} \sigma^{2}(T-\tau) S^{2} V_{S S}+\frac{1}{2} \sigma^{2}(T-\tau) V_{X X}+\sigma^{2}(T-\tau) S V_{S X}+\bar{\beta}(T-\tau)(\psi(T-\tau)+X- \\
\xi(T-\tau) \ln S) S V_{S}+(\mu(T-\tau)+\sigma(T-\tau) \hat{\theta}(T-\tau)-\alpha(T-\tau) X) V_{X}-\rho V+ \\
\lambda(T-\tau) \int_{\mathbb{R}}\left(V\left(S e^{\eta(T-\tau) z}, X, I, \tau\right)-V(S, X, I, \tau)-S\left(e^{\eta(T-\tau) z}-1\right) V_{S}\right) e^{\tilde{\theta} z} \bar{l}(d z)+ \\
\max _{c \in C(I)}\left\{(c-a(I, c)) S-(c+a(I, c)) V_{I}\right\},
\end{gathered}
$$

with the initial condition

$$
V(S, X, I, 0)=H(S, I, X)
$$

The domain of the PIDE (33) is $S \times X \times I=[0, \infty) \times(-\infty, \infty) \times\left[0, I_{\max }\right]$. But we will restrict our analysis to the finite domain $\left[0, S_{\max }\right) \times\left(X_{\min }, X_{\max }\right) \times\left[0, I_{\max }\right]$.

When $S \rightarrow 0$, (33) simplifies to

$$
\begin{array}{r}
V_{\tau}=\frac{1}{2} \sigma^{2}(T-\tau) V_{X X}+(\mu(T-\tau)+\sigma(T-\tau) \hat{\theta}(T-\tau)-\alpha(T-\tau) X) V_{X}- \\
\rho V+\max _{c \in C(I)}\left\{-(c+a(I, c)) V_{I}\right\},
\end{array}
$$

For the limit $S \rightarrow S_{\max }$ we apply $V_{S S} \rightarrow 0$ and $V_{S X} \rightarrow 0$ to get

$$
\begin{gathered}
V_{\tau}=\frac{1}{2} \sigma^{2}(T-\tau) V_{X X}+\bar{\beta}(T-\tau)(\psi(T-\tau)+X- \\
\left.\xi(T-\tau) \ln S_{\max }\right) S_{\max } V_{S}+(\mu(T-\tau)+\sigma(T-\tau) \hat{\theta}(T-\tau)-\alpha(T-\tau) X) V_{X}-\rho V+ \\
\lambda(T-\tau) \int_{\mathbb{R}}\left(V\left(S_{\max } e^{\eta(T-\tau) z}, X, I, \tau\right)-V\left(S_{\max }, X, I, \tau\right)-S_{\max }\left(e^{\eta(T-\tau) z}-1\right) V_{S}\right) e^{\tilde{\theta} z} \bar{l}(d z)+ \\
\max _{c \in C(I)}\left\{(c-a(I, c)) S_{\max }-(c+a(I, c)) V_{I}\right\},
\end{gathered}
$$

We can get the boundary conditions for the limits $X \rightarrow X_{\min }$ and $X \rightarrow X_{\max }$ in a similar way by assuming that $V_{X X} \rightarrow 0$ and $V_{S X} \rightarrow 0$. We need to look at the joint $\operatorname{limits}(S, X) \rightarrow\left(0, X_{\min }\right),(S, X) \rightarrow\left(S_{\max }, X_{\max }\right),(S, X) \rightarrow\left(0, X_{\max }\right)$ and $(S, X) \rightarrow$ $\left(S_{\max }, X_{\min }\right)$ as well in order to extend our boundary conditions to the corner points. 
For instance, the first joint limit will result in the following condition on the corner point $\left(0, X_{\min }\right)$ :

$$
\begin{aligned}
& V_{\tau}=\left(\mu(T-\tau)+\sigma(T-\tau) \hat{\theta}(T-\tau)-\alpha(T-\tau) X_{\min }\right) V_{X}- \\
& \rho V+\max _{c \in C(I)}\left\{-(c+a(I, c)) V_{I}\right\},
\end{aligned}
$$

The corresponding equations for the other 3 corners can be derived in a similar way.

Now, let's take the change of variable $y=\ln S$ and define:

$$
V\left(e^{y}, X, I, t\right)=\bar{V}(y, X, I, t) \quad \text { and } \quad H\left(e^{y}, X, I\right)=h(y, X, I) .
$$

Then our PIDE simplifies to

$$
\begin{gathered}
\bar{V}_{\tau}=\frac{1}{2} \sigma^{2}(T-\tau)\left(\bar{V}_{y y}+\bar{V}_{x x}\right)+\sigma^{2}(T-\tau) \bar{V}_{x y}+\left(-\frac{1}{2} \sigma^{2}(T-\tau)+\bar{\beta}(T-\tau)(\chi(T-\tau)+x-\right. \\
\left.\xi(T-\tau) y)) \bar{V}_{y}+(\mu(T-\tau)+\sigma(T-\tau) \hat{\theta}(T-\tau)-\alpha(T-\tau) x) \bar{V}_{x}\right)-\rho \bar{V}+ \\
\int_{\mathbb{R}}\left(\bar{V}(y+\eta(T-\tau) z, x, I, \tau)-\bar{V}(y, x, I, \tau)-\left(e^{\eta(T-\tau) z}-1\right) \bar{V}_{y}\right) \lambda^{\mathbb{Q}}(T-\tau) P_{J}^{\mathbb{Q}}(d z)+ \\
\max _{c \in C(I)}\left\{(c-a(I, c)) e^{y}-(c+a(I, c)) \bar{V}_{I}\right\}, \quad(34)
\end{gathered}
$$

with the initial condition

$$
\bar{V}(y, x, I, 0)=h(y, x, I)
$$

Denote

$$
\begin{aligned}
& \bar{\gamma}(\tau)=\lambda^{\mathbb{Q}}(T-\tau) \int_{\mathbb{R}}\left(e^{\eta(T-\tau) z}-1\right) P_{J}^{\mathbb{Q}}(d z), \\
& \gamma(\tau)=\chi(T-\tau)-\frac{\bar{\gamma}(\tau)}{\bar{\beta}(T-\tau)} .
\end{aligned}
$$

Then (34) becomes

$$
\begin{gathered}
\bar{V}_{\tau}=\frac{1}{2} \sigma^{2}(T-\tau)\left(\bar{V}_{y y}+\bar{V}_{x x}\right)+\sigma^{2}(T-\tau) \bar{V}_{x y}+(\bar{\beta}(T-\tau)(\gamma(\tau)+x- \\
\left.\left.\xi(T-\tau) y)-\frac{1}{2} \sigma^{2}(T-\tau)\right) \bar{V}_{y}+(\mu(T-\tau)+\sigma(T-\tau) \hat{\theta}(T-\tau)-\alpha(T-\tau) x) \bar{V}_{x}\right)- \\
\left(\rho+\lambda^{\mathbb{Q}}(T-\tau)\right) \bar{V}+\lambda^{\mathbb{Q}}(T-\tau) \int_{\mathbb{R}}\left(\bar{V}(y+\eta(T-\tau) z, x, I, \tau) P_{J}^{\mathbb{Q}}(d z)+\right. \\
\max _{c \in C(I)}\left\{(c-a(I, c)) e^{y}-(c+a(I, c)) \bar{V}_{I}\right\},
\end{gathered}
$$

with the initial condition

$$
\bar{V}(y, x, I, 0)=h(y, x, I) \quad \text { for } \quad \forall x, y \in \mathbb{R} .
$$

Now, we need to transform our boundary conditions defined earlier in this section. The bounded domain of the equation $(35)$ is $y \times x \times I=\left[y_{\min }, y_{\max }\right] \times\left[x_{\min }, x_{\max }\right] \times\left[0, I_{\max }\right]$. 
So, the boundary conditions for $S \rightarrow 0$ and $S \rightarrow S_{\max }$ become

$$
\begin{aligned}
& \bar{V}_{\tau}=\frac{1}{2} \sigma^{2}(T-\tau) \bar{V}_{x x}+(\mu(T-\tau)\left.+\sigma(T-\tau) \hat{\theta}(T-\tau)-\alpha(T-\tau) x) \bar{V}_{x}\right) \\
&-\left(\rho+\lambda^{\mathbb{Q}}(T-\tau)\right) \bar{V}+\max _{c \in C(I)}\left\{-(c+a(I, c)) \bar{V}_{I}\right\}
\end{aligned}
$$

and

$$
\begin{gathered}
\bar{V}_{\tau}=\frac{1}{2} \sigma^{2}(T-\tau) \bar{V}_{x x}+\left(\bar{\beta}(T-\tau)\left(\gamma(\tau)+x-\xi(T-\tau) y_{\max }\right)-\frac{1}{2} \sigma^{2}(T-\tau)\right) \bar{V}_{y}+ \\
\left.(\mu(T-\tau)+\sigma(T-\tau) \hat{\theta}(T-\tau)-\alpha(T-\tau) x) \bar{V}_{x}\right)-\left(\rho+\lambda^{\mathbb{Q}}(T-\tau)\right) \bar{V}+ \\
\lambda^{\mathbb{Q}}(T-\tau) \int_{\mathbb{R}}\left(\bar{V}\left(y_{\max }+\eta(T-\tau) z, x, I, \tau\right) P_{J}^{\mathbb{Q}}(d z)+\right. \\
\max _{c \in C(I)}\left\{(c-a(I, c)) e^{y_{\max }}-(c+a(I, c)) \bar{V}_{I}\right\},
\end{gathered}
$$

when $y \rightarrow y_{\min }$ and $y \rightarrow y_{\max }$, respectively. The other boundary equations transform in a similar way. We will discuss the requirements on the coefficients for each boundary condition in the following section.

\section{Discretisation}

The PIDE (35) can be written as

$$
\bar{V}_{\tau}=\mathcal{D} \bar{V}+\mathcal{H} \bar{V}+\max _{c \in C(I)}\left\{(c-a(I, c)) e^{y}-(c+a(I, c)) \bar{V}_{I}\right\}
$$

where

$$
\begin{aligned}
& \mathcal{D} \bar{V}=\frac{1}{2} \sigma^{2}(T-\tau)\left(\bar{V}_{y y}+\bar{V}_{x x}\right)+\sigma^{2}(T-\tau) \bar{V}_{x y}+(\bar{\beta}(T-\tau)(\gamma(\tau)+x- \\
& \left.\left.\xi(T-\tau) y-\frac{1}{2} \sigma^{2}(T-\tau)\right)\right) \bar{V}_{y}+(\mu(T-\tau)+\sigma(T-\tau) \hat{\theta}(T-\tau)- \\
& \alpha(T-\tau) x) \bar{V}_{x}-\left(\rho+\lambda^{\mathbb{Q}}(T-\tau)\right) \bar{V}
\end{aligned}
$$

and

$$
\mathcal{H} \bar{V}=\lambda^{\mathbb{Q}}(T-\tau) \int_{\mathbb{R}}\left(\bar{V}(y+\eta(T-\tau) z, x, I, \tau) P_{J}^{\mathbb{Q}}(d z) .\right.
$$

We will extend the explicit finite difference method proposed in [25] to solve our nonlinear PIDE (38) with two state variables, time-dependent coefficients and seasonal jump intensity. First of all, we need to restrict our PIDE from $\mathbb{R}$ to bounded domain:

$$
\mathbb{R} \rightarrow]-A, A[\text {, where } A>0
$$

and impose an artificial boundary condition w.r.t. $y$ at all points outside $]-A, A[$ :

$$
\bar{V}(y, x, I, \tau)=h(y, x, I), \quad \text { for } \quad \forall y \notin]-A, A[.
$$


We then replace the domain $[-A, A] \times[-A, A] \times\left[0, I_{\max }\right] \times[0, T]$ by the following discrete grid:

$$
\begin{aligned}
\tau_{n} & =n \Delta \tau, \quad n=0, \ldots, M, \quad \Delta \tau=\frac{T}{M}, \\
y_{i} & =-A+i \Delta y, \quad i=0, \ldots, N, \quad \Delta y=\frac{2 A}{N}, \\
x_{u} & =-A+u \Delta x, \quad u=0, \ldots, R, \quad \Delta x=\frac{2 A}{R}, \\
I_{j} & =j \Delta I, \quad j=0, \ldots, L, \quad \Delta I=\frac{I_{\max }}{L} .
\end{aligned}
$$

So, $\bar{V}\left(y_{i}, x_{u}, I_{j}, \tau_{n}\right)=\bar{V}(i \Delta y, u \Delta x, j \Delta I, n \Delta \tau)$ is the exact solution of the PIDE at the node $\left(y_{i}, x_{u}, I_{j}, \tau_{n}\right)$, while $\bar{V}_{i, u, j}^{n}$ is the approximate solution at the same node.

For the integral term we need some finite upper/lower bounds $B_{u}, B_{l}$ to truncate the large jumps. So, the integral terms become

$$
\begin{aligned}
& \int_{B_{l}}^{B_{u}}\left(\bar{V}(y+\eta(T-\tau) z, x, I, \tau) P_{J}^{\mathbb{Q}}(d z),\right. \\
& \bar{\gamma}(\tau)=\lambda^{\mathbb{Q}}(T-\tau) \int_{B_{l}}^{B_{u}}\left(e^{\eta(T-\tau) z}-1\right) P_{J}^{\mathbb{Q}}(d z)
\end{aligned}
$$

To approximate the integral terms we can use the trapezoidal quadrature rule where $\Delta z=\Delta y$. To avoid any interpolation issues we introduce the following change of variable:

$$
z^{\prime}=y+\eta(T-\tau) z \Longrightarrow z=\frac{z^{\prime}-y}{\eta(T-\tau)} \Longrightarrow d z=\frac{d z^{\prime}}{\eta(T-\tau)}
$$

Let take $K_{l}, K_{u}$ such that $\left[B_{l}, B_{u}\right] \subset\left[\left(K_{l}-\frac{1}{2}\right) \Delta y,\left(K_{u}+\frac{1}{2}\right) \Delta y\right]$. Then we have

$$
\begin{gathered}
\int_{B_{l}}^{B_{u}}\left(\bar{V}(y+\eta(T-\tau) z, x, I, \tau) P_{J}^{\mathbb{Q}}(d z)=\right. \\
\int_{y_{i}+\eta\left(T-\tau_{n}\right) B_{l}}^{y_{i}+\eta\left(T-\tau_{n}\right) B_{u}}\left(\bar{V}\left(z^{\prime}, x_{u}, I_{j}, \tau_{n}\right) P_{J}^{\mathbb{Q}}\left(\frac{d z^{\prime}}{\eta(T-\tau)}\right) \approx \sum_{s=K_{l}}^{K_{u}} \bar{V}_{s, u, j}^{n} P_{s}\right. \\
\bar{\gamma}\left(\tau_{n}\right) \approx \hat{\gamma}\left(\tau_{n}\right)=\lambda^{\mathbb{Q}}\left(T-\tau_{n}\right) \sum_{s=K_{l}}^{K_{u}}\left(e^{\eta\left(T-\tau_{n}\right) z}-1\right) P_{s}(d z)
\end{gathered}
$$

where

$$
P_{s}=\int_{\left(s-\frac{1}{2}\right) \Delta y}^{\left(s+\frac{1}{2}\right) \Delta y} P_{J}^{\mathbb{Q}}\left(\frac{d z^{\prime}}{\eta(T-\tau)}\right) .
$$

Contrary to [25], we assume that $\lambda$ is a given deterministic function of $t$ and therefore, we don't need to derive it numerically. We need to enlarge our domain to $\left[-A+B_{l}, A+B_{u}\right]$ 
in order to compute the integral terms. Thus, the discretised grid w.r.t. $y$ extends from $i=K_{l}$ to $i=K_{u}+N$, where $\bar{V}_{i, u, j}^{n}=h\left(y_{i}, x_{u}, I_{j}\right)$ for $i \notin[0, N]$. Let us denote

$$
\begin{aligned}
A(\tau, y, x) & =\bar{\beta}(T-\tau)(\gamma(\tau)+x-\xi(T-\tau) y)-\frac{1}{2} \sigma^{2}(T-\tau), \\
B(\tau, x) & =\mu(T-\tau)+\sigma(T-\tau) \hat{\theta}(T-\tau)-\alpha(T-\tau) x
\end{aligned}
$$

and therefore

$$
\begin{aligned}
A_{i, u}^{n} & =A\left(\tau_{n}, y_{i}, x_{u}\right), \\
B_{u}^{n} & =B\left(\tau_{n}, x_{u}\right) .
\end{aligned}
$$

to simplify the ensuing numerical work. Note that we need to choose the coefficients in such a way that $\lim _{y \rightarrow y_{\min }} A(\tau, x, y) \geq 0, \lim _{y \rightarrow y_{\max }} A(\tau, x, y) \leq 0, \lim _{x \rightarrow x_{\text {min }}} B(\tau, x) \geq$, and $\lim _{x \rightarrow x_{\max }} B(\tau, x) \leq$,0 . If we have these conditions then no other information is required from outside the domain to solve the equation on the corresponding boundary. We can now apply finite difference formulas to approximate the partial derivatives appearing in the PIDE and boundary conditions:

$$
\begin{aligned}
\left(\bar{V}_{y}\right)_{i, u, j}^{n} \approx\left\{\begin{array}{lll}
\frac{\bar{V}_{i+1, u, j}^{n}-\bar{V}_{i, u, j}^{n},}{\Delta y}, & \text { if } & A_{i, u}^{n} \geq 0 \\
\frac{\bar{V}_{i, u, j}^{n}-V_{i-1, u, j}^{n}}{\Delta y}, & \text { if } & A_{i, u}^{n}<0,
\end{array}\right. \\
\left(\bar{V}_{x}\right)_{i, u, j}^{n} \approx\left\{\begin{array}{lll}
\frac{\bar{V}_{i, u+1, j}^{n}-\bar{V}_{i, u, j}^{n},}{\Delta x}, & \text { if } & B_{u}^{n} \geq 0 \\
\frac{\bar{V}_{i, u, j}^{n}-\bar{V}_{i, u-1, j}^{n}}{\Delta x}, & \text { if } & B_{u}^{n}<0,
\end{array}\right. \\
\left(\bar{V}_{y y}\right)_{i, u, j}^{n} \approx \frac{\bar{V}_{i+1, u, j}^{n}-2 \bar{V}_{i, u, j}^{n}+\bar{V}_{i-1, u, j}^{n}}{\Delta y^{2}}, \\
\left(\bar{V}_{x x}\right)_{i, u, j}^{n} \approx \frac{\bar{V}_{i, u+1, j}^{n}-2 \bar{V}_{i, u, j}^{n}+\bar{V}_{i, u-1, j}^{n}}{\Delta x^{2}}, \\
\left(\bar{V}_{x y}\right)_{i, u, j}^{n} \approx \frac{\bar{V}_{i+1, u+1, j}^{n}-\bar{V}_{i+1, u-1, j}^{n}-\bar{V}_{i-1, u+1, j}^{n}+\bar{V}_{i-1, u-1, j}^{n}}{4 \Delta x \Delta y}, \\
\left(\bar{V}_{\tau}\right)_{i, u, j}^{n} \approx \frac{\bar{V}_{i, u, j}^{n+1}-\bar{V}_{i, u, j}^{n}}{\Delta \tau} .
\end{aligned}
$$

Then our diffusion part becomes

$$
\begin{aligned}
&(D \bar{V})_{i, u, j}^{n} \approx \frac{1}{2} \sigma^{2}\left(T-\tau_{n}\right)\left(\frac{\bar{V}_{i+1, u, j}^{n}-2 \bar{V}_{i, u, j}^{n}+\bar{V}_{i-1, u, j}^{n}}{\Delta y^{2}}+\frac{\bar{V}_{i, u+1, j}^{n}-2 \bar{V}_{i, u, j}^{n}+\bar{V}_{i, u-1, j}^{n}}{\Delta x^{2}}\right)+ \\
& \sigma^{2}\left(T-\tau_{n}\right) \frac{\bar{V}_{i+1, u+1, j}^{n}-\bar{V}_{i+1, u-1, j}^{n}-\bar{V}_{i-1, u+1, j}^{n}+\bar{V}_{i-1, u-1, j}^{n}}{4 \Delta x \Delta y}+ \\
& A_{i, u}^{n} \frac{\bar{V}_{i+1, u, j}^{n}-\bar{V}_{i, u, j}^{n}}{\Delta y}+B_{u}^{n} \frac{\bar{V}_{i, u+1, j}^{n}-\bar{V}_{i, u, j}^{n}}{\Delta x}-\left(\rho+\lambda^{\mathbb{Q}}\left(T-\tau_{n}\right) \bar{V}_{i, u, j}^{n}, \quad\right.
\end{aligned}
$$

where we have assumed that $A_{i, u}^{n} \geq 0$ and $B_{u}^{n} \geq 0$. The terms $-\bar{V}_{i+1, u-1, j}^{n}$ and $-\bar{V}_{i-1, u+1, j}^{n}$ will cause problems by damaging the monotonicity of the finite difference scheme. [11] 
suggests adding a corrector to the above diffusion part in the following way to solve this issue:

$$
(D \bar{V})_{i, u, j}^{n}+\frac{\sigma^{2}\left(T-\tau_{n}\right)}{4 \Delta x \Delta y} \Delta_{y}^{+} \Delta_{y}^{-} \Delta_{x}^{+} \Delta_{x}^{-} \bar{V}_{i, u, j}^{n}
$$

where

$$
\begin{array}{rlrl}
\Delta_{y}^{+} \bar{V}_{i, u} & =\bar{V}_{i+1, u}-\bar{V}_{i, u}, & \Delta_{x}^{+} \bar{V}_{i, u}=\bar{V}_{i, u+1}-\bar{V}_{i, u}, \\
\Delta_{y}^{-} \bar{V}_{i, u}=\bar{V}_{i, u}-\bar{V}_{i-1, u}, & \Delta_{x}^{-} \bar{V}_{i, u}=\bar{V}_{i, u}-\bar{V}_{i, u-1} .
\end{array}
$$

If we omit $j$ just for now and take $\Delta x=\Delta y$ then (41) can be rewritten as:

$$
\begin{aligned}
& \frac{\sigma^{2}\left(T-\tau_{n}\right)}{2 \Delta y^{2}}\left(\bar{V}_{i+1, u+1}^{n}-2 \bar{V}_{i, u}^{n}+\bar{V}_{i-1, u-1}^{n}\right)+ \\
& \frac{A_{i, u}^{n}}{\Delta y}\left(\bar{V}_{i+1, u}^{n}-\bar{V}_{i, u}^{n}\right)+\frac{B_{u}^{n}}{\Delta y}\left(\bar{V}_{i, u+1}^{n}-\bar{V}_{i, u}^{n}\right)-\left(\rho+\lambda^{\mathbb{Q}}\left(T-\tau_{n}\right)\right) \bar{V}_{i, u}^{n},
\end{aligned}
$$

where $n=1, \ldots, M, i=K_{l}+1, \ldots, K_{u}-1$ and $u=1, \ldots N-1$. The corrected scheme (42) is now monotone. After denoting the approximate value of $c\left(y_{i}, I_{j}, \tau_{n}\right)$ by $\omega_{i, j}^{n}$ our explicit scheme becomes

$$
\begin{aligned}
\frac{\bar{V}_{i, u, j}^{n+1}-\bar{V}_{i, u, j}^{n}}{\Delta \tau}= & D \bar{V}_{i, u, j}^{n}+H \bar{V}_{i, u, j}^{n}+ \\
& \max _{\omega_{i, j}^{n} \in C(I)}\left\{\left(\omega_{i, j}^{n}-a\left(I_{j}, \omega_{i, j}^{n}\right)\right) e^{y_{i}}-\left(\omega_{i, j}^{n}+a\left(I_{j}, \omega_{i, j}^{n}\right)\right) \frac{\bar{V}_{i, u, j+1}^{n}-\bar{V}_{i, u, j}^{n}}{\Delta I}\right\}
\end{aligned}
$$

where

$$
\begin{gathered}
D \bar{V}_{i, u, j}^{n}=\frac{\sigma^{2}\left(T-\tau_{n}\right)}{2 \Delta y^{2}}\left(\bar{V}_{i+1, u+1}^{n}-2 \bar{V}_{i, u}^{n}+\bar{V}_{i-1, u-1}^{n}\right)+ \\
\frac{A_{i, u}^{n}}{\Delta y}\left(\bar{V}_{i+1, u}^{n}-\bar{V}_{i, u}^{n}\right)+\frac{B_{u}^{n}}{\Delta y}\left(\bar{V}_{i, u+1}^{n}-\bar{V}_{i, u}^{n}\right)-\left(\rho+\lambda^{\mathbb{Q}}\left(T-\tau_{n}\right) \bar{V}_{i, u}^{n}\right. \\
H \bar{V}_{i, u, j}^{n}=\lambda^{\mathbb{Q}}\left(T-\tau_{n}\right) \sum_{s=K_{l}}^{K_{u}} \bar{V}_{s, u, j}^{n} P_{s}
\end{gathered}
$$

When $\left(\left(\omega_{i, j}^{n}+a\left(I_{j}, \omega_{i, j}^{n}\right)\right)>0\right.$ we will discretise $\bar{V}_{I}$ with $\frac{\bar{V}_{i, u, j}^{n}-\bar{V}_{i, u, j-1}^{n}}{\Delta I}$. We know from the initial condition that $\bar{V}_{i, u, j}^{0}=h\left(y_{i}, x_{u}, I_{j}\right)$. So, starting from time $\tau_{0}=0$ we first solve the maximization problem

$$
\hat{\omega}_{i, j}^{n}=\underset{\omega_{i, j}^{n} \in C(I)}{\arg \max }\left\{\left(\omega_{i, j}^{n}-a\left(I_{j}, \omega_{i, j}^{n}\right)\right) e^{y_{i}}-\left(\omega_{i, j}^{n}+a\left(I_{j}, \omega_{i, j}^{n}\right)\right) \frac{\bar{V}_{i, u, j+1}^{n}-\bar{V}_{i, u, j}^{n}}{\Delta I}\right\},
$$


then substitute this optimal strategy $\hat{\omega}_{i, j}^{n}$ in $(44)$ to get

$$
\begin{aligned}
\bar{V}_{i, u, j}^{n+1}=\bar{V}_{i, u, j}^{n}+\Delta \tau D \bar{V}_{i, u, j}^{n}+\Delta \tau H \bar{V}_{i, u, j}^{n}+ \\
\Delta \tau\left\{\left(\hat{\omega}_{i, j}^{n}-a\left(I_{j}, \hat{\omega}_{i, j}^{n}\right)\right) e^{y_{i}}-\left(\hat{\omega}_{i, j}^{n}+a\left(I_{j}, \hat{\omega}_{i, j}^{n}\right)\right) \frac{\bar{V}_{i, u, j+1}^{n}-\bar{V}_{i, u, j}^{n}}{\Delta I}\right\}
\end{aligned}
$$

where $n=0,1, \ldots, M-1$.

\section{Numerical results}

In this section, we conduct a numerical experiment based on the discretisation scheme presented in the previous sections. As the default units for gas spot price $S$ and gas inventory $I$, we will use "dollars per million British thermal unit" $(\$ / \mathrm{mmBtu})$ and million cubic feet (MMcf), respectively. Note that $1000 \mathrm{mmBtus}$ are roughly equal to $1 \mathrm{MMcf}$. Therefore, we need to multiply gas spot price by 1000 when computing payos or revenues in order to unify the units.

For better understanding of the problem and tracking of the results, we will start the section by the analysis of the simple case with no jumps and seasonality. In other words, our underlying model is given by one factor geometric mean-reverting model. We will then apply the spikes and seasonality of the spot price and jump-size. We will assume that other parameters of the underlying spot price dynamics are constant as it will not affect the generality of the results. Meanwhile, we will refer to [26] for the definition of the gas loss rate $a(I, c)$ :

$$
a(I, c)=a(c)= \begin{cases}0 & \text { for } \quad c \geq 0 \\ 1.7365 & \text { for } \quad c<0\end{cases}
$$

which means that gas loss occurs on a fixed rate $1.7 \mathrm{MMcf} /$ day only when we inject gas into the storage. In this case we can explicitly solve the optimisation part of the equation (38):

$$
c=\left\{\begin{array}{lcc}
c_{\max }(I) & \text { for } & 1000 P>V_{I} \\
c_{\text {min }}(I) & \text { for } & \left(c_{\text {min }}(I)-1.7365\right) 1000 P>\left(c_{\text {min }}(I)+1.7365\right) V_{I} \\
0 & & \text { otherwise }
\end{array}\right.
$$

Note that there was a mistake in this formula in the original paper which has been corrected here. The payoff at maturity can be defined in different ways. Based on [26] and [13] we can use $V(S, X, I, T)=0$ or $V(S, X, I, T)=-2 S \max \{1000-I, 0\}$. In this work, we look at the second case in order to compare our results with [13].

Let's first look at the following model without jumps and seasonality:

$$
\frac{d S(t)}{S(t)}=\beta(\chi-\xi \ln S(t)) d t+\sigma d B^{\theta}(t)
$$

By taking the parameters as $\beta=3.4, \chi=\log 6, \xi=1, \sigma=0.59$ and $\rho=0.1$ our PIDE 


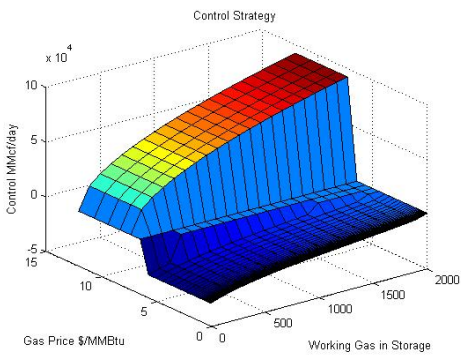

(a)

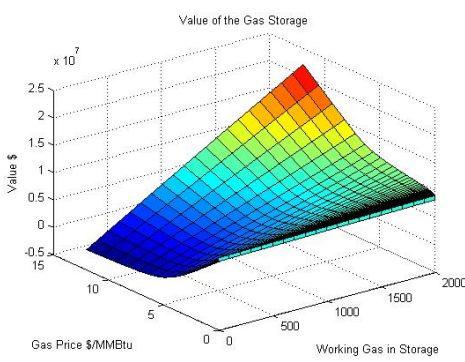

(b)

Figure 1.: Control strategy (a) and value (b) surfaces for the one factor model (48).

Table 1.: Input parameters used to evaluate the gas storage contract

\begin{tabular}{|l|l|l|l|}
\hline Parameter & Value & Parameter & Value \\
\hline$I_{\max }$ & $2000 \mathrm{MMcf}$ & $k_{2}$ & 730000 \\
\hline$T$ & 3 years & $k_{3}$ & 500 \\
\hline$k_{1}$ & 2040.41 & $k_{4}$ & 2500 \\
\hline
\end{tabular}

(33) transforms to the following PDE:

$$
\begin{aligned}
V_{\tau}=\frac{1}{2} 0.59^{2} S^{2} V_{S S}+3.4(\log 6-\ln S) S V_{S}-0.1 V+ \\
\max _{c \in C(I)}\left\{1000(c-a(c)) S-(c+a(c)) V_{I}\right\} .
\end{aligned}
$$

The rest of the parameters required to price the gas storage contract is given in Table 1 . The maximum value that the spot price can achieve is $S_{\max }=2000 \$ / \mathrm{mmBtu}$. As we are using an explicit scheme, the number of time steps needs to be large enough to get stable results. So, we will take 8000, 40 and 20 grid nodes for the variables $\tau, S$ and $I$, respectively.

The control strategies for operating the gas storage facility are presented in Figure 1(a). We can distinguish three visible regions:negative, zero and positive. We do nothing in the zero region, while negative and positive areas correspond to injecting gas into and releasing it from the storage, respectively. Figure 1(a) implies that gas can be pumped into the reservoir very quickly when gas inventory levels are low. The injectivity rate gets less deeper and approaches to 0 as working gas in the storage increases. In contrast, the deliverability rate increases as the level of gas gets higher. We can also observe that the zero region is thicker in high inventory levels as it gets more expensive to inject gas when the reservoir is almost full.

Figure 1(b) depicts the value surface of the contract. We can see that the facility gets its lowest value when prices are high and the reservoir is empty and approaches to its highest value when the storage is full and the prices are high.

After investigating our numerical approach for the one factor model, we can move to solving difference equations (45). We will keep parameters presented for the one factor model the same. The additional parameters are $\mu=\log 5$ and $\alpha=1$. The surfaces of control strategy and contract value obtained from the numerical implementation for a 


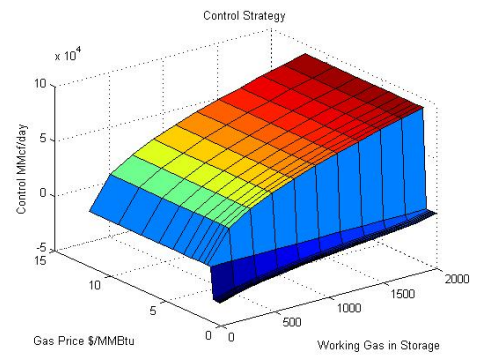

(a) Control strategy

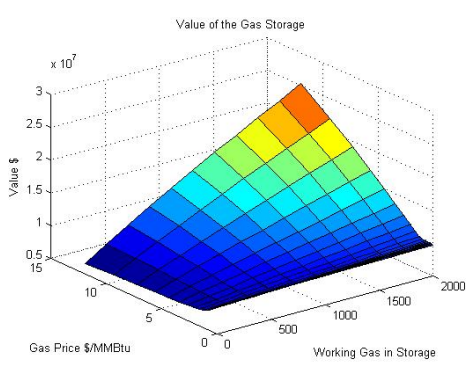

(b) Value surface

Figure 2.: Control strategy and value surfaces for the PIDE (38) at the fixed $X=-1.86$

fixed $x=-1.86$ are shown in Figure 2. According to Figure 2(a) the zero region of no action is thinner than in Figure 1(a). That is why, the value goes up to its maximum more rapidly as $S$ increases (Figure $2(\mathrm{~b})$ ).

\section{Conclusion and future research plans}

In this research we analysed a stochastic control problem for natural gas storage facilities while taking into account operating characteristics. The real option based valuation approach by [26] was combined with the spot price dynamics presented by [6]. The key differences of this two-factor spot price model from the original one are instant spikes with a time-dependent jump intensity. The resulting non-linear HJB equation was solved by an explicit finite difference method. The numerical approach gives us both the value of the storage contract and optimal operating strategy depending on the gas price, current level of gas in the storage and time. The monotonicity issue of the scheme was solved by using the corrector of the diffusion term of the PIDE. The numerical method was implemented in MATLAB for different underlying models. The effects of seasonality and seasonal jump intensity were analysed by observing the surfaces of control strategies and contract values. As the next step, empirical analysis of natural gas spot prices needs to be conducted in order to fit our model to the data. We plan to follow [5] by using a normal inverse Gaussian (NIG) distribution to model the residuals of time series. It is also possible to extend the NIG Lévy process to the generalised hyperbolic model (see [16]) in order to obtain a better fitting to the data.

It may be interesting to extend this control model to include regime-switching in natural gas spot prices like in [14] while taking into account independent increment property. The application of the optimisation approach into gas-fired and multi-fuel power plant operations could also be investigated. 


\section{Bibliography}

[1] O.E. Barndorff-Nielsen and N. Shephard, Non-Gaussian Ornstein-Uhlenbeck-based models and some of their uses in financial economics, Journal of the Royal Statistical Society: Series B (Statistical Methodology) 63 (2001), pp. 167-241.

[2] F.E. Benth, The stochastic volatility model of Barndorff-Nielsen and Shephard in commodity markets, Mathematical Finance 21 (2011), pp. 595-625.

[3] F.E. Benth, J.S. Benth, and S. Koekebakker, Stochastic modelling of electricity and related markets, Vol. 11, World Scientific, 2008.

[4] F.E. Benth, J. Kallsen, and T. Meyer-Brandis, A non-Gaussian Ornstein-Uhlenbeck process for electricity spot price modeling and derivatives pricing, Applied Mathematical Finance 14 (2007), pp. 153-169.

[5] F.E. Benth and J. ŠALTYTE-BENTH, The normal inverse Gaussian distribution and spot price modelling in energy markets, International journal of theoretical and applied finance 7 (2004), pp. 177-192.

[6] F.E. Benth and C. Sgarra, The risk premium and the Esscher transform in power markets, Stochastic Analysis and Applications 30 (2012), pp. 20-43.

[7] F.E. Benth, L. Vos, et al., Pricing of forwards and options in a multivariate non-Gaussian stochastic volatility model for energy markets, Advances in Applied Probability 45 (2013), pp. 572-594.

[8] P. Bjerksund, G. Stensland, and F. Vagstad, Gas storage valuation: Price modelling v. optimization methods (2008).

[9] A. Boogert and C. De Jong, Gas storage valuation using a Monte Carlo method, The journal of derivatives 15 (2008), pp. 81-98.

[10] A. Boogert and C. de Jong, Gas storage valuation using a multifactor price process, The Journal of Energy Markets 4 (2011), pp. 29-52.

[11] F. Bouchut and H. Frid, Finite difference schemes with cross derivatives correctors for multidimensional parabolic systems, Journal of Hyperbolic Differential Equations 3 (2006), pp. 27-52.

[12] R. Carmona and M. Ludkovski, Valuation of energy storage: An optimal switching approach, Quantitative Finance 10 (2010), pp. 359-374.

[13] Z. Chen and P.A. Forsyth, A semi-Lagrangian approach for natural gas storage valuation and optimal operation, SIAM Journal on Scientific Computing 30 (2007), pp. 339-368.

[14] Z. Chen and P.A. Forsyth, Implications of a regime-switching model on natural gas storage valuation and optimal operation, Quantitative Finance 10 (2010), pp. 159-176.

[15] A.K. Dixit, Investment under uncertainty, Princeton university press, 1994.

[16] E. Eberlein and U. Keller, Hyperbolic distributions in finance, Bernoulli (1995), pp. 281-299.

[17] A. Eydeland and K. Wolyniec, Energy and power risk management: New developments in modeling, pricing, and hedging, Vol. 206, John Wiley \& Sons, 2003.

[18] J. Gray and P. Khandelwal, Towards a realistic gas storage model, Commodities Now (2004), pp. $1-4$.

[19] J.D. Hamilton, Analysis of time series subject to changes in regime, Journal of econometrics 45 (1990), pp. 39-70.

[20] P. Henaff, I. Laachir, and F. Russo, Gas storage valuation and hedging. A quantification of the model risk, arXiv preprint arXiv:1312.3789 (2013).

[21] J. Jacod and A. Shiryaev, Limit theorems for stochastic processes, Vol. 288, Springer Science \& Business Media, 2013.

[22] F.A. Longstaff and E.S. Schwartz, Valuing American options by simulation: A simple least-squares approach, Review of Financial studies 14 (2001), pp. 113-147.

[23] C. Parsons, Quantifying natural gas storage optionality: a two-factor tree model, Journal of Energy Markets 6 (2013), pp. 95-124.

[24] E.S. Schwartz, The stochastic behavior of commodity prices: Implications for valuation and hedging, The Journal of Finance 52 (1997), pp. 923-973.

[25] P. Tankov, Financial modelling with jump processes, CRC press, 2003.

[26] M. Thompson, M. Davison, and H. Rasmussen, Natural gas storage valuation and optimization: A real options application, Naval Research Logistics (NRL) 56 (2009), pp. 226-238.

[27] X. Warin, Gas storage hedging, in Numerical Methods in Finance, Springer, 2012, pp. 421-445. 